\title{
El impacto de las redes sociales en el proceso de enseñanza aprendizaje de la termodinámica
}

\section{The impact of social networks in the teaching process of thermodynamics}

RANGEL-ROMERO, Carlos $\dagger^{*}$, ROJAS-GARNICA, Juan Carlos, MORENO-GUZMÁN, Javier Andrey y HERNÁNDEZ-LAZCANO, Ricardo

Universidad Tecnológica de Puebla

ID $1^{\mathrm{er}}$ Autor: Carlos, Rangel-Romero / ORC ID: 0000-0003-4879-4228, CVU CONACYT ID: 894477

ID $1^{\text {er }}$ Coautor: Juan Carlos, Rojas-Garnica / ORC ID: 0000-0002-2261-587X, CVU CONACYT ID: 66417

ID $2^{\text {do }}$ Coautor: Javier Andrey, Moreno-Guzmán / ORC ID: 0000-0002-3510-2213, CVU CONACYT ID: 266302

ID $3^{\text {er }}$ Coautor: Ricardo, Hernández-Lazcano / ORC ID: 0000-0003-1142-2930, CVU CONACYT ID: 920171

DOI: $10.35429 / J I T .2019 .21 .6 .26 .30$

Recibido: 12 de Agosto, 2019; Aceptado 30 de Diciembre, 2019

\section{Resumen}

El desarrollo y la utilización de las redes sociales en el proceso de enseñanza aprendizaje que desarrollan los alumnos, hace que se convierta en una herramienta importante y casi imprescindible en el proceso educativo. Esto conlleva cambios, de diversas características absolutamente inevitables cuando se tratan de poner en marcha propuestas innovadoras en la enseñanza de la termodinámica. De ahí que los procesos formativos en las universidades demanden nuevos modelos de estrategia pedagógica capaces de responder a las necesidades de la nueva sociedad. Sin embargo, en la práctica, durante el proceso de enseñanza aprendizaje de la termodinámica se ha detectado que aún se asumen enfoques tradicionalistas de enseñanza lo que no satisface las expectativas que demandan los nuevos modelos de formación. La investigación que se lleva a cabo tiene como objeto de estudio la evaluación del proceso de enseñanza aprendizaje en su formación formativa y significativa, teniendo como objetivo propiciar la evaluación formativa a través del uso de los entornos virtuales de formación.

Termodinámica, Redes Sociales, Entornos virtuales de formación

\begin{abstract}
The development and use of social networks in the teaching-learning process that students develop, makes it an important and almost essential tool in the educational process. This entails changes, of various absolutely inevitable characteristics when it comes to launching innovative proposals in the teaching of thermodynamics. Hence the training processes in universities demand new models of pedagogical strategy capable of responding to the needs of the new society. However, in practice, during the teaching process of thermodynamics, it has been detected that traditionalist teaching approaches are still assumed, which does not meet the expectations demanded by the new training models. The research carried out is aimed at studying the evaluation of the teaching-learning process in its formative and meaningful training, with the objective of promoting formative evaluation through the use of virtual training environments.
\end{abstract}

Thermodynamics, Social Networks, Virtual training environments

Citación: RANGEL-ROMERO, Carlos, ROJAS-GARNICA, Juan Carlos, MORENO-GUZMÁN, Javier Andrey y HERNÁNDEZLAZCANO, Ricardo. El impacto de las redes sociales en el proceso de enseñanza aprendizaje de la termodinámica. Revista de Tecnologías de la Información. 2019. 6-21: 26-30

\footnotetext{
* Correspondencia del Autor: (carlos.rangel@utpuebla.edu.mx)

$\dagger$ Investigador contribuyendo como primer autor.
} 


\section{Introducción}

Actualmente, las redes sociales desarrollan un gran impacto sobre el conocimiento que generan los alumnos en las instituciones de educación superior ya que se generan innovaciones en los procesos de enseñanza-aprendizaje. Referiendonos específicamente a la utilización de los entornos virtuales en la modalidad educativa se sabe que cada vez son utilizados con mayor frecuencia para que los alumnos comprueben los fenómenos, observen el funcionamiento de las máquinas, $\mathrm{y}$, en ocasiones, encontrar explicaciones más claras y diferentes a lo que los docentes les proporcionan, todo esto lo pueden encontrar en: aulas virtuales, videoconferencias, correo electrónico, chats, foros y bibliotecas digitales.

De este modo, se despliega un lenguaje cotidiano en el contexto universitario, logrando pertinencia y calidad en la incorporación de tecnologías de información y comunicación. De ahí que los nuevos enfoques educativos deberán promover la creación de ambientes de aprendizaje que permitan el desarrollo de un clima propicio para la generación de un conocimiento que cumplan con las condiciones actuales que los alumnos necesitan, con el único fin de promover el aprendizaje activo.

Un punto importante en el empleo de las redes sociales sobre el aprendizaje de la termodinámica lo constituyen las tutorías o asesorías académicas presenciales que se realizan cara a cara, pero al ser alternadas con el uso de medios y herramientas de aprendizaje, como los entornos virtuales, originan cambios en la didáctica y en el rol del docente, mejorando la praxis pedagógica al crear estrategias significativas en los procesos de enseñanza y aprendizaje.

Dentro de las innovaciones educativas, la incorporación de los entornos virtuales de aprendizaje implica dar un vuelco al proceso de adquisición y transformación del conocimiento; de allí que la pertinencia, calidad y utilidad educativa consiste en saber integrar las actividades de aprendizajes significativos y aprovechar las herramientas que ofrecen los entornos virtuales. De ahí la importancia de rediseñar, adecuar el currículo y adaptarlo a los nuevos cambios tecnológicos, mediante la construcción de una estructura pedagógica y metodológica fundamentada en contenidos, evaluación y experiencias.
Cabe señalar que a pesar de los avances tecnológicos, todavía su incorporación en el ambiente educativo no se ha generalizado, posiblemente por la falta de compromiso institucional y por la resistencia de los docentes debido, quizás, a la idea errada de ser desplazados por la tecnología, así como por la falta de recursos para la inversión en infraestructuras y talento humano calificado.

Es importante mencionar que actualmente las redes sociales tienen un gran impacto en la vida de los estudiantes universitarios. Por tal razón los profesores nos enfrentamos día a día ante la disyuntiva de utilizarlas o no como una herramienta didáctica más, con el objetivo de acercarnos a su realidad, ya que es innegable que el uso de redes sociales constituye, según algunos estudios realizados una parte importante de su vida cotidiana. La presente investigación pretende dar a conocer las ventajas y desventajas del uso de las redes sociales en el proceso de enseñanza aprendizaje de la materia de termodinámica.

El trabajo de investigación que se utilizó fue la metodología de recolección de datos, con instrumentos tanto cualitativos (Grupo focal), como cuantitativos (encuesta). Finalmente, podemos afirmar que si bien es cierto las horas dedicadas en éstas, pudieran considerarse una pérdida de tiempo o distractor inminente en el aprendizaje de nuestros alumnos, también nos brindan un área de oportunidad en el desarrollo de una didáctica efectiva para los docentes en la mejora de la calidad educativa y los procesos de enseñanza aprendizaje.

La presente investigación sobre las redes sociales como un elemento importante en la inclusión digital, en la cual se pretende buscar las posibilidades de transformación profesional, en donde el docente requiere analizar y reconocer su entorno con respecto a la evolución de las Tecnologías de la Información y la Comunicación (TIC) dentro de las exigencias que se tienen. 


\section{Desarrollo}

Actualmente es muy importante que los docentes sean capaces de interrelacionarse con sus alumnos sin barreras de espacio y tiempo. Ya que el nuevo paradigma de las redes sociales en el proceso de enseñanza aprendizaje aporta elementos que permiten situar a la educación en un terreno más propicio que en el pasado para elaborar procesos de aprendizaje más sencillos mediante modelos educativos más adaptados al ser humano, a partir de un mejor conocimiento de su identidad.

Se hace posible ofrecer a los estudiantes de cualquier edad el gusto, el entrenamiento, los medios para observar, interrogar, buscar y estudiar sobre cualquier tema de su interés de manera más profunda, así como brindarles las facilidades y oportunidades para investigar, preguntar, dialogar $\mathrm{y}$ emitir sus propias conclusiones, logrando con ello que el alumno genere su propio conocimiento de acuerdo a sus necesidades y que este conocimiento adquirido pueda ser sometida al juicio de sus propios compañeros y profesores El profesor por otra parte debe evolucionar y crecer junto con sus alumnos, apartándose de la educación tradicional donde se pensaba que él era el poseedor del conocimiento y era el único capaz de transmitirlo.

Siempre será importante tener en cuenta que educar es despertar la curiosidad, animar, estimular tanto la aptitud interrogativa como el autodidactismo y orientar hacia los problemas fundamentales de nuestra condición y de nuestra época. Por otra parte siempre se tendrá que tener en cuenta que los problemas a los que enfrentamos actualmente con los alumnos son, la violencia, ausencia de tolerancia, la falta de respeto hacia los demás y hacia nosotros mismos, por lo que como docentes no debemos permitir que siga creciendo esa ola de destrucción y corrupción, que, para el caso de este estudio, se observa que a través de las redes sociales se llega a trasgredir la integridad de las personas, con comentarios mal intencionados; lo cual nos lleva a pensar que se hace un uso negativo de las redes sociales y por lo que vemos un área de oportunidad para encauzar el uso de redes sociales hacia cuestiones positivas como el aprendizaje colaborativo.
En este sentido el nuevo Paradigma plantea que en lugar de insistir en el pensamiento analítico, lineal del hemisferio izquierdo, se tratará de educar el cerebro en su totalidad e insistir en la fusión de los dos procesos: el hemisferio derecho y sus estrategias holísticas, no lineales e intuitivas. En vez de aumentar nuestra confianza en la tecnología y en los conocimientos dispensados por el profesor, a menudo obsoletos, se subrayará la importancia de las relaciones humanas y de la enseñanza mutua, a través de diversos medios educativos. Pudiendo considerar a las redes sociales, por el auge que tienen actualmente con los jóvenes, un medio que como docentes debiéramos adecuar en el ámbito de la educación para tratar de alcanzar la finalidad planteada por el Nuevo Paradigma de la Educación.

Nos encontramos entonces, ante la gran interrogante de lograr fortalecer o no, el aprendizaje y las actitudes positivas anteriormente mencionadas, a través de un canal de comunicación propio, sencillo y real de nuestros estudiantes ( $\mathrm{y}$ distinto al aula tradicional): Las redes sociales. Esto por un lado, y por otro, nos encontramos ante las voces que fríamente señalan que las redes sociales son solo modas, simples espacios virtuales que propician la pérdida de tiempo, el ocio y malos hábitos. Encontramos incluso docentes que las satanizan y las señalan abiertamente como interruptores o distractores innecesarios deshumanizadores.

\section{Análisis de Resultados}

El fenómeno de las redes sociales en el proceso de enseñanza aprendizaje se ha entrometido en los alumnos para generar su propio conocimiento en diferentes formas, con la creación de YouTube (video), Instagram (fotografía), Twitter, entre otras, sin embargo, los objetivos siempre serán exactamente los mismos que en el pasado y que tendrán en el futuro, esto es, fomentar las relaciones entre los individuos para cualquier fin, únicamente cambiando las formas. El uso de las redes sociales ha sido un fenómeno social a nivel mundial muy fuerte permitiendo demostrar las ventajas y desventajas que a esto conlleva y sobre todo permitiendo generar muchas ideas que pueden ser implementadas de manera local en nuestros centros de trabajo para aprovechar las bondades de estas, reduciendo la brecha digital e incrementando la inclusión digital en las personas, no importando las condiciones sociales, políticas, culturales y geográficas.

RANGEL-ROMERO, Carlos, ROJAS-GARNICA, Juan Carlos, MORENO-GUZMÁN, Javier Andrey y HERNÁNDEZ-LAZCANO, Ricardo. El impacto de las redes sociales en el proceso de enseñanza aprendizaje de la termodinámica. Revista de Tecnologías de la Información. 2019 
De las actividades que se utilizan en el uso de las redes sociales se destacan los envíos de mensajes privados, compartir fotos, foros, videos, notas y juegos, todo esto se puede realizar bajo la misma red social en la mayoría de ellas por lo que es posible que se dedique el mismo tiempo dedicado a estas actividades. Otro dato que se arrojó es que la mayoría de los encuestados comentan haber dejado de hacer algunas actividades por seguir dedicándole tiempo a las redes sociales, entre las que destaca el estudio, mencionando que la que más utilizan es Facebook.

Por lo que fue necesario enfatizar en la utilización de redes sociales en la educación y con una escala de 5 a 10 se les preguntó la frecuencia de uso de las redes en actividades académicas por semana a lo que se obtuvo lo siguiente, los estudiantes universitarios utilizan las redes sociales para la solución de dudas en los contenidos temáticos o para los exámenes, también para complementar lo visto en clase, entrega de trabajos, intercambio de apuntes, documentos e información, obtener información sobre la vida universitaria, organizar actividades extra académicas, consultar con expertos en temas particulares, tutorías y consultas con sus profesores.

Es importante mencionar que las redes sociales y el uso de la tecnología en la docencia va en auge ya que estas son muy útiles creando una comunicación más estrecha entre los alumnos y el profesor, además que agiliza el proceso de compartir información de manera rápida entre los usuarios, pero estas deben requerir un análisis de la metodología educativa para que se obtenga un mayor beneficio en el uso de estas.

Comprobando que se favorezca el trabajo colaborativo y si la comunicación mejora tanto entre los alumnos. Se menciona que las redes sociales se vuelven cada vez más atractivas si el número de usuarios aumenta, así que entre más sean los alumnos y maestros que participen mayor será el éxito del ambiente de trabajo, con gran libertad y sin restricciones, donde los usuarios serán responsables de respetar a sus demás compañeros y profesores así como a la institución.
El impacto en el uso de las redes sociales se verá reflejada en las ventajas sobre la comunicación e integración a grupos sociales en un mismo espacio, la cooperación será un punto indispensable para la elaboración o desarrollo de proyectos o actividades educativas, con el uso de las redes se logrará crear grupos de trabajo ya sean abiertos o cerrados y se facilitará la coordinación entre ellos, pues esto permitiría abordar temas de interés o de estudio, así como tutorías con una comunicación más directa entre alumnos o con sus profesores, por medio de mensajes privados, foros, blogs, etc. Donde el alumno encontrará una gran cantidad de información a su disposición en cualquier momento y los profesores tomarán el papel de apoyo y guía para los alumnos en su aprendizaje, todo esto permitiendo el uso eficaz de las TIC's.

\section{Agradecimientos}

Se agradece las facilidades prestadas para la elaboración del presente trabajo a la Universidad Tecnológica de Puebla.

\section{Conclusiones}

La versatilidad de las tecnologías ha transformado profundamente la sociedad y ha iniciado una revolución irreversible en la educación. Especialmente ha invertido el paradigma pedagógico que giraba entorno a la escuela, centro tradicional de atracción y punto esencial de aprendizaje. Las redes sociales han impactado de tal forma que han permitido que la distribución del conocimiento se realice dentro y fuera del aula, llevándola hacia el hogar y el trabajo, gracias al empleo creciente de la informática y las telecomunicaciones. Sin embargo, el docente debe vigilar el uso adecuado de las redes sociales para evitar: que circule información sin sustento y que sea puente para agresiones a los miembros de los grupos.

\section{Trabajo Futuro}

En un próximo trabajo se hace indispensable registrar de manera sistemática las evidencias que nos permitan analizar y evaluar los avances obtenidos.

\section{Referencias}

[1] García J. (2011). Modelo educativo basado en competencias: Importancia y necesidad. Vol.11, No.3. pp.1-24. 
[2] Filmus, D. (1994). El papel de la Educación frente a los desafíos de las transformación científico-tecnológicas. Publicado con autorización de los editores. Presentado Volumen II, Módulo V del Curso de Formación de Administradores de la Educación, publicado en Buenos Aires por el Ministerio de Cultura y Educación de la República Argentina, la Facultad Latinoamericana de Ciencias Sociales (FLACSO-Buenos Aires) y la Organización de Estados Iberoamericanos para la Educación, la Ciencia y la Cultura (OEI), del libro "Para qué sirve la escuela". Tesis. Grupo editorial Norma. Buenos Aires. Recuperado el 28 de febrero de 2011.

[3] Ibáñez, C. (2007). Diseño curricular basado en competencias profesionales: una propuesta desde la psicología interconductual. Revista de Educación y Desarrollo, Número XIX (6). Recuperado el 27 de Febrero de 2011

[4] Alarcón, R. (2014). Conferencia Inaugural Universidad 2014, 9no Congreso Internacional de Educación Superior. La Habana: MES.

[5] Estrada, John. (2012). La formación por competencias y el mundo del trabajo: de la calificación a la empleabilidad. Revista Salud pública, (1). Pp.98-111.

[6] Fernández, Eduardo. (2009). El discurso de la formación basada en competencias profesionales. Un análisis crítico de la formación inicial de Profesionales en la Educación Superior. REIFOP, (1), PP.: 151-160.

[7] Del Rey, A.; Sánchez, J. (2011). Crítica de la educación por competencias. Universitas. (15). Pp. 233-246. Revista de Ciencias Sociales y Humanas.

[8] AZZURRO, A.; Girón, P. y Cura, R.O. (2014). "Articulación entre docencia e investigación de la enseñanza en entorno industrial". En VIII Congreso Iberoamericano de Docencia Universitaria y Educación Superior. Rosario, Universidad Nacional de Ros 